\title{
Die Vater-Sohn-Beziehung im autobiografischen Roman Als Vaters Bart noch rot war - Ein Roman in Geschichten von Wolfdietrich Schnurre
}

\author{
Çiğdem Kırca (D), Samsun- Birkan Kargı (D), Amasya \\ https://dx.doi.org/10.37583/diyalog.759414
}

\begin{abstract}
Deutsch)
In Gedenken an seinen 100. Geburtstag widmet sich die vorliegende Arbeit einem bedeutenden Lyriker und Erzähler der deutschen Nachkriegsliteratur. Wolfdietrich Schnurre wies in seinen Werken auf die Schuldproblematik der Nachkriegsgesellschaft hin. Seine Erzählungen schildern die Verhältnisse der NSZeit sowie deren Auswirkungen auf die autoritäre Gesellschaft. Er leistete mit seinen Werken einen großen Beitrag bei der Demokratisierung als auch Aufarbeitung der Vergangenheit bei. Auch in seinem autobiografischen Roman Als Vaters Bart noch rot war - Ein Roman in Geschichten befasste sich Schnurre mit der Nachkriegsgesellschaft und ihren Problematiken. Den Schwierigkeiten der Zeit und den politischen sowie sozialen Veränderungen sind Vater und Sohn ausgesetzt, die sie zusammen zu überwältigen versuchen. Die Vater-Sohn-Beziehung in ausgewählten Geschichten des Romans ist Untersuchungsgegenstand der Arbeit. Anhand der hermeneutischen und werkimmanenten Methode soll aufgezeigt werden, welche Problematiken Schnurre in seinen Geschichten anspricht und wie er diese überwältigt, in dem er Vater und Sohn zusammen agieren lässt. Merkmalspezifische Handlungsweisen von Vater und Sohn sollen aus pädagogischem Blickwinkel betrachtet werden, um im Anschluss zwischen den Handlungsweisen und der damaligen autoritären Gesellschaft einen Bogen spannen zu können.
\end{abstract}

Schlüsselwörter: Nachkriegsliteratur, Schuldproblematik, autoritäre Gesellschaft, autobiografischer Roman, Hermeneutik

\section{Abstract (English)}

The Father-Son Relationship in the Autobiographical Novel Als Vaters Bart noch rot war - Ein Roman in Geschichten by Wolfdietrich Schnurre

In memory of his $100^{\text {th }}$ birthday, the present work is dedicated to an important poet and narrator of postwar German literature. Wolfdietrich Schnurre is one of the most important poets and storytellers in postwar German literature. Schnurre pointed out the problem of guilt in post-war society in his works. His stories describe the conditions of the Nazi era and their effects on society. With his works, he made a great contribution to democratization as well as reappraisal of the past. Also in his autobiographical novel Als Vaters Bart noch rot war - Ein Roman in Geschichten Schnurre, he dealt with post-war society and its problems. The difficulties of the time and the political and social changes are exposed to father and son, who try to overcome them together. The father-son relationship in selected stories from the novel is the aim of this study. The hermeneutic and inherent method is intended to show which problems Schnurre 
addresses in his stories and how he overcomes them by allowing father and son to act together. Characteristic actions of father and son should be viewed from a pedagogical point of view in order to be able to bridge the gap between the actions and the authoritarian society at that time.

Keywords: Post-war literature, debt problems, authoritarian society, autobiographical novel, hermeneutic

\section{EXTENDED ABSTRACT}

In commemoration of his $100^{\text {th }}$ birthday, the year 2020 is particularly suitable for literary contributions to Wolfdietrich Schnurre and his works. He was an author who preferred to covertly record the dark side of humanity, as in his work Der Schattenfotograf. In his works, he pointed out the problems of guilt in post-war society. Through a socially critical and poetic spelling, the contemporary problems of the readers were brought to the eyes. Wolfdietrich Schnurre saw in writing the only acceptable form of atonement. It was important to him to be aware of the guilt, even in the place of those who did not want to be aware of their own guilt. For this reason, he made a great contribution to democratization with his works. His stories describe the conditions of the Nazi era and their effects on society, and made it possible to deal with the past. Also in his autobiographical novel Als Vaters Bart noch rot war - ein Roman in Geschichten Schnurre, he dealt with post-war society and its problems, which is the subject of the present study. The first part of the work is devoted to the problem of guilt. What was Wolfdietrich Schnurre's fault? In order to give an answer to this question and to be able to establish links between the autobiographical novel and his life, his sense of art has been taken into account. In the second part of the study, the father-son relationship is examined in selected stories from a pedagogical point of view, in order to subsequently be able to establish a link between the way of acting and the authoritarian society at that time. The analysis of the problem of guilt in the stories was the focus of the study, i.e. in what way do father and son overcome the problems arising from authoritarian society? The last part of the study tries to reconcile Wolfdietrich Schnurre's understanding of the artist with the literary characteristics of his stories that he had previously developed.

Wolfdietrich Schnurre, as an artist, saw his task and the purpose of his writing in finding the burden of life's guilt in all truth. The reader should be given the opportunity to deal with the problem of guilt in his works. The autobiographical novel Als Vaters Bart noch rot war is the first volume of the planned two volumes and was first published in 1958. The second volume, titled Als Vater sich den Bart abnahm, was published by his wife Marina Schurre from his estate. The father-son stories take place in Berlin in the twenties and early thirties. At a time when people were affected by the Great Depression, the beginning of National Socialism and the Second World War was still ahead of them. The discourse run in the stories between father and son and is told from the perspective of the son Bruno, in a simple and naive narrative. The stories deal with destinies, problems of ordinary people that can even be called outsiders because of their prevailing situation. The stories Jenö war mein Freund - Der Bröchtenclou - Die 
Leihgabe served as the subject of the analysis. After a brief summary of the respective stories, the core of the socio-political problem was brought together. Subsequently, the ways of overcoming the identified socio-political problems were examined in the light of the father-son relationship. There are three plot lines, which are repeated in the stories. The starting point of the stories is initially a difficult, often financial emergency situation in which the father and son find themselves. The authoritarian pressure of society follows this emergency situation. This authoritarian pressure serves as a drive to act, i.e. he lets the characters act contrary to the contemporary situation. Finally, the father and the son overcome the socio-political problem through cohesion, mutual understanding and empathy.

Two faces are presented to the reader, which in turn carry another one. The father bears the face of the past and the son bears the face of the future. This duality can be summarized under the Janus motif. The past symbolizes the end of something and the future the beginning, but without the past, there would be no future or vice versa. Because of this complementary relationship, the future and the past must be viewed as a whole. The pedagogically correct behavior of the father does not help him and his son to swim, but rather to swim against the stream of authoritarian society. Wolfdietrich Schnurre's stories can be described as timeless because they deal with social issues in a sociocritical way, which also exist and will continue to exist. A real work of art can never be interpreted to the end and this also includes the works of Wolfdietrich Schnurre. 


\section{Einleitung}

Schreiben ist für mich die

einzig akzeptable Form der Sühne

Mit diesem Zitat beschreibt Wolfdietrich Schnurre den Grund für sein Schreiben. Es schildert die für ihn wichtige komplementäre Beziehung zwischen Schuld und Schreiben. Dieses Zitat stammt aus einem Antwortbrief an Walter Kolbenhoff. In ihrem Briefwechsel diskutieren die beiden Autoren über den Künstler und sein Werk sowie seine Stellung zur Gegenwart und seinen Problemen. Der Briefwechsel erschien in der Zeitschrift Der Skorpion, an der neben Schnurre auch andere Schriftsteller und Publizisten mitarbeiteten. Im September 1947 versammelten sich diese, um die Zeitschrift vorzubereiten. Schnurre, der die legändere Tagung mit seiner bekannten Erzählung Das Begräbnis eröffnete, war somit auch Mitglied der Gruppe 47, die bei dieser Versammlung entstand. Für ihn war die Schreiberei kein Weg die „Last der Lebensschuld $\mathrm{zu}$ verringern, sondern sie hat die Aufgabe, sie unbestechlich aufzuzeigen, in aller Wahrhaftigkeit immer wieder auf sie hinzuweisen." (Richter 1948: 45). Sein Schreiben hatte nicht das Ziel sein Gewissen zu reinigen, sondern die Aufgabe sich der Schuld bewusst zu werden, sich dazu zu bekennen. Woran aber bestand die Schuld von Wolfdietrich Schnurre? Um dieser Fragestellung eine Antwort geben und Knüpfpunkte zwischen dem autobiografischen Roman und Autor herstellen zu können, wird zunächst im ersten Teil dieser Arbeit sein Leben sowie Künstlerverständnis betrachtet. Der zweite Teil der Arbeit widmet sich dem autobiografischen Roman Als Vaters Bart noch rot war - Ein Roman in Geschichten (1958). Aus pädagogischem Blickwinkel soll die Vater-Sohn-Beziehung in ausgewählten Geschichten untersucht werden, um im Anschluss einen Bogen zwischen der Handlungsweise und der damaligen autoritären Gesellschaft herstellen zu können. Die Aufarbeitung der Schuldproblematik in den Geschichten stand im Vordergrund der Arbeit, d.h. in welcher Art und Weise überwinden Vater und Sohn die Problematiken, die aufgrund der autoritären Nachkriegsgesellschaft entstehen? Der letzte Teil der Arbeit versucht das Künstlerverständnis von Wolfdietrich Schnurre mit den zuvor erarbeiteten literarischen Merkmalen seiner Geschichten in Einklang zu bringen. Es soll aufgezeigt werden, wie sich die Last der Lebensschuld in aller Wahrhaftigkeit wiederfinden lässt, denn darin sah Wolfdietrich Schnurre seine Aufgabe als Künstler und den Sinn seines Schreibens. Dem Leser sollte die Möglichkeit der Auseinandersetzung mit der Schuldproblematik in seinen Werken gegeben werden, denn ,einer, der sich schuldig fühlt, und tapfer die Unsühnbarkeit seiner Schuld erkannt hat, [lebt] sauberer und aufrechter als einer, dem das alles noch unklar ist." (Richter 1948: 45).

\section{Wolfdietrich Schnurre - Leben und Künstlerverständnis}

Die neue deutsche Literatur nach 1945 wurde mit dem mehrdeutigen Etikett Trümmerliteratur gezeichnet, sie verdankt nach Schnurre ,ihr Entstehen keinem 
organischen Wachstum [...] sondern einer Katastrophe“ (Lüdemann 1975: 149). Neben Autoren wie Heinrich Böll und Wolfgang Borchert gehörte auch Wolfdietrich Schnurre zu denen, die vom Krieg gebrandmarkt wurden und sich mit den Kriegsfolgen, dem Nationalsozialismus sowie der Schuldproblematik in ihren Werken auseinandersetzen. Am 22. August 1920 wurde Schnurre als Sohn eines Bibliothekars in Frankfurt am Main geboren und verstarb er am 09. Juni 1989 in Kiel. Zu seiner Lebenszeit war er bis 1945 als Soldat im Dienst, danach als Kritiker und freier Schriftsteller tätig. ,Schnurre erlebt das Dasein als ein Gefangener des genormten und mechanisierten Lebens in der Gegenwart" (Brauneck 1995: 700). Als Humanist und Friedensbefürworter behandelte Schnurre in seinen Werken Probleme der Kriegs- und Nachkriegszeit auf eine satirische-zeitkritische Weise, indem er den vereinsamten Menschen in seiner Ausweglosigkeit darstellte und den Menschen eine bessere Zukunft verhoffen lässt (Karg1 2009: 25). Als Wehrmachtssoldat gehörte Schnurre zunächst zu den Eifrigen, "die gar nicht gehorchensselig und "tapfer" genug sein konnten" (Richter 1948: 43). Doch nach ungefähr vier Jahren begann es in ihm wie bei einem Trümmerfall zu bröckeln. Während seiner Zeit als Soldat wurde er aufgrund Befehlsverweigerungen abermals inhaftiert. Einen wichtigen Abschnitt in seinem Leben nahm die legendäre Gruppe 47 ein. Schnurre war Mitglied der Gruppe 47, deren Hauptziel Mitbegründer Hans Werner Richter wie folgt erklärt:

\footnotetext{
War die >Gruppe $47<$ einmal eine Art Werkstatt gewesen, so war sie jetzt ein überdimensionales Lektorat. Der Autor las nun nicht mehr anderen Autoren seine Arbeit vor, um ihre Meinung und unter Umständen ihren Rat zu hören, er las sie nunmehr auch vor einer geschlossenen Gruppe von Berufskritikern, die schnell, eloquent, oft geistreich witzig und immenser Sachkenntnis das Vorgelesene unter die Lupe nahmen, es hin und her wendeten, von dieser und von jener Seite beäugten, und es behandelten wie einen mehr oder weniger kostbaren Edelstein, der durch etwas besseren Schliff noch gewinnen konnte, oder auch wie einen Gegenstand, der ihrer Aufmerksamkeit nicht würdig sei (Bohn 1995: 247).
}

Viele bekannte Autoren haben ihren Ruhm durch die Mitgliedschaft der Gruppe 47 erlangt und umgekehrt wurde sie wiederum durch die Autoren bekannt. Grund für die Bildung der literarischen Gruppe war die Zeitschrift Der Skorpion, deren Veröffentlichung angestrebt war und neben ihrem Begründer Hans Werner Richter auch andere Autoren wie Alfred Andersch, Walter Kolbenhoff, Wolfgang Bächler, Günter Eich und darunter auch Wolfdietrich Schnurre mitwirkte. 1967 wurde die Gruppe 47 von Richter einberufen und die geplante Veröffentlichung der Zeitschrift fand auch nie statt. Doch sie war der eigentliche Grund für ,eine demokratische Elitenbildung auf dem Gebiet der Literatur und der Publizistik [...] ohne Programm, ohne Verein, ohne Organisation und ohne irgendeinem kollektiven Denken Vorschub zu leisten“ (Lüdemann 1975: 150). Dies sind die Worte von Richter über die Gruppe 47, die ihren Namen ihrem Gründungsjahr 1947 verdankte. Die Tagungen dienten als eine Art Plattform, auf der für die Zeitschrift verfassten Manuskripte vorgelesen und strenger Kritik unterzogen wurden. Schnurre las seine Geschichte Das Begräbnis, die den 
Seelenzustand einer aus dem Krieg heimkehrenden jungen Generation schildert, beim erstmaligen als auch letzten Treffen der Gruppe 47 vor und aus diesem Grund symbolisiert die Geschichte den Anfang und das Ende der legendären Gruppe 47 (Bauer 1996: 60 f.). Er arbeitete als Theater- und Filmkritiker für verschiedene Zeitungen wie den Horizont, die Deutsche Rundschau sowie Neue Zeitung und war nach 1950 als freier Schriftsteller tätig (Ernst 1993: 1226). Den Grund für sein Schreiben sah er in seinem Schuldbewusstsein und als Folge daraus beim Bedürfnis des Helfen- und Klärenwollens sowie in der ,bohrende[n] Unruhe, die heut ja jeden schöpferischen Geist gepackt hat, über das Chaos auf der Welt, von dem wir nicht wissen: wird es ein Anfang oder wird es ein Ende sein“ (Richter 1948:45). In diesen Zeilen kristallisiert sich auch sein Verständnis von einem Künstler. Sich der Schuld über die Vergangenheit bewusst sein, denen Helfen die es noch nicht sind und über zukünftige Ereignisse warnen, darin sah er als schöpferischer Geist, d.h. als Künstler seine Berufung. An dieser Stelle lassen sich die oftmals zitierten Verse von Schnurre (1948) anführen, die „auf poetische Weise gegen die Poesie“ zu schreiben auffordert (Barner 1994: 77).

\author{
zerschlagt eure Lieder \\ verbrennt eure Verse \\ sagt nackt \\ was ihr müßt.
}

Sein Mitwirken als Soldat im Krieg und die Kriegserlebnisse der Zeit belasteten ihn wie auch andere Schriftsteller seelisch zu tiefst. Die nackte Wahrheit, wie sie auch bereits Schnurre in seinen Versen abverlangte, ließ sich mit der Lyrik zur Sprache bringen. Erlebnisse und Erfahrungen, Eindrücke und Empfindungen der Nachkriegszeit konnten mit ihr zum Ausdruck gebracht werden, weshalb sie auch als Trümmerlyrik bezeichnet wird.

\footnotetext{
Die Trümmerlyrik der Nachkriegszeit [wird] zum Ort einer Diskussion, in der es um Vergangenheit, Gegenwart und Zukunft geht, eine Diskussion, die schwankt zwischen Untergangsstimmungen und der Euphorie des Aufbruchs, zwischen Depression und Zukunftsgewissenheit, zwischen Resignation und Optimismus (Schnell 2001: 494).
}

Die Schriftsteller der Zeit schrieben damals aus Notwendigkeit und Unvermeidlichkeit in einer einfachen, klaren und konkreten Sprache, was sich aufgrund der vorherrschenden propagandistischen Sprache als schwierig gestalten ließ, da sie von der nationalsozialistischen Emphase und dem rhetorischen Pathos geprägt war (Schnell 2001: 494). $\mathrm{Zu}$ dieser Zeit befand sich in Deutschland die Kurzgeschichte als literarische Form in ihrer Blütezeit. Dem amerikanischen Vorbild short story entsprechend "sucht sie die Menschen in ihrem Alltag auf, aber in lebenswichtigen Stunden, in Augenblicken der Entscheidung zwischen Gut und Böse, zwischen Leben und Tod" (Lüdemann 1975: 27). Auch Schnurre nutzte oftmals die Kurzgeschichte als 
literarische Form, um seiner Aufgabe als Künstler gerecht zu werden. In ihnen befasste er sich mit dem Schuldmotiv, der Nachkriegsgesellschaft und den Druckmechanismen der Nationalsozialisten und nannte die Dinge in seinen Werken beim Namen. Dies tat er auf eine ironische und verfremdete Weise, in dem er zwischen den erwarteten und verwirklichten Geschehnissen eine Konfrontation wahr werden lässt, die zu einer Spannungssteigerung beim Leser führen, die wiederum aufgrund der vorherrschenden rationalen und emotionalen Konfliktsituation ihnen die Möglichkeit einer Distanzierung gibt und somit sie zu einer kognitiven Produktion zwingt (Karg1 2009: 28). 1982 erhielt Schnurre den Kölner Literaturpreis, 1983 den Büchner-Preis und 1989 den Kieler Kulturpreis (Brauneck 1995: 700).

\section{Die Vater-Sohn-Geschichten von Wolfdietrich Schnurre}

In dem autobiografisch geprägten Buch Als Vaters Bart noch rot war - Ein Roman in Geschichten werden die Vater-Sohn-Geschichten zusammengestellt, die mit Wolfdietrich Schnurre sofort in Verbindung gebracht werden. Dieser erste von den geplanten zwei Bändern ist erstmalig 1958 beim Arche Verlag erschienen. Der zweite Band, welcher den Titel Als Vater sich den Bart abnahm trägt, wurde von seiner Ehefrau Marina Schnurre aus seinem Nachlass herausgegeben. Die zwanzig in sich geschlossenen Vater-Sohn-Geschichten spielen in Berlin in den zwanziger und anfangenden dreißiger Jahren ab. In einer Zeit, in der die Menschen von der Weltwirtschaftskrise, von dem beginnenden Nationalsozialismus betroffen waren und ihnen noch der Zweite Weltkrieg bevorstand. Die Handlungsstränge verlaufen in den Geschichten zwischen Vater und Sohn und werden aus der Perspektive des Sohnes Bruno erzählt. Wobei an dieser Stelle erwähnt werden muss, dass Schnurre sich selbst mit dieser Figur identifiziert und mit der Vaterfigur Otto in den Geschichten seinen eigenen Vater darstellt. In einem Brief an seine Schülerin (1963) wird der autobiografische Strang zwischen den Geschichten und Schnurres Leben deutlich.

\footnotetext{
[...] weil ich mit Bruno, dem kleinen Jungen, der es erzählt, identisch bin und die Zeit und den Zeithintergrund damals aus eigener Anschauung kenne. Und der Vater schließlich, die eigentliche Hauptfigur, der ist auch wirklich vorhanden, und er lebt auch Gott sei Dank noch, in Berlin [...] (Hirschenauer/Weber 1970: 7).
}

Ort des Geschehens ist wie bereits schon erwähnt wurde Berlin, die Stadt in der damals auch Schnurre unter der Obhut seines Vaters aufgewachsen ist. Nachdem sich seine Eltern getrennt hatten, lebte Schnurre nämlich mit seinem Vater zusammen, was auch gleichzeitig der Grund dafür ist, dass in den Geschichten keine Mutter als Leitfigur zu finden ist, denn diese fehlte auch in seiner eigenen Kindheit. Seine „Kindheitseindrücke hat Schnurre in seinen Vater-Sohn-Geschichten literarisch gestaltet.“ (Bauer 1996: 100).

Aufgrund seines Erfahrungshorizontes beschrieb Schnurre sehr realistisch und detailgetreu die Ecken und Winkel nicht nur der Berliner Gassen und Straßen, sondern auch die Ecken und Kanten der Leute. In einer Reportage erklärt Marina Schnurre 
warum genau deshalb das Buch und seine Geschichten bislang noch aktuell und zeitlos sind.

\begin{abstract}
Also das kann ich Ihnen sagen. Ich bin mit diesen Geschichten in der Hand sozusagen Ost-Berlin abgelaufen, weil er hat ja realistisch alle Straßen auch beschrieben, in denen er da aufgewachsen ist mit seinem Vater, dort gelebt hat, und Sie können es als Buch nehmen und heute noch diese Ecken alle finden. Und heute noch haben wir diese selbe Lage: Die Leute sind arbeitslos, es geht ihnen nicht so gut. Er hat immer gerne kleine Leute beschrieben, und die treffen Sie heute noch dort. Und insofern ist dieses Buch, auch der zweite Band, immer noch ganz aktuell (Kassel 2010).
\end{abstract}

Die Geschichten sind in einer einfachen Sprache verfasst und behandeln Schicksäle, Probleme von kleinen Leuten, die man sogar aufgrund ihrer Situation als Außenseiter bezeichnen kann. Nach einer detaillierten Analyse der im Sammelband enthaltenden Kurzgeschichten wurden drei exemplarisch ausgewählt, um Wiederholungen zu vermeiden. Die Kurzgeschichten Jenö war mein Freund; Der Brötchenclou und Die Leihgabe wurden als Untersuchungsgegenstand gewählt, da sie in der Weltliteratur einen festen Platz besitzen und die Verbundenheit zwischen Vater und Sohn, d.h. die Dualität hervorbringt. Im Folgenden sollen in den ausgewählten Geschichten die VaterSohn Beziehung untersucht werden.

\title{
3.1 Jenö war mein Freund
}

Diese Geschichte handelt über eine Freundschaft mit einem Zigeunerjungen, die bereits nach kurzer Zeit auf tragische Weise endet. Wie bereits aus der Überschrift entnommen werden kann, war sein Name Jenö. Bruno und Jenö freundeten sich beim Grasrupfen auf dem Stadion am Faulen See an. Obwohl beide damals noch Kinder waren, Bruno war 9 und Jenö 8 Jahre alt, genossen sie beide eine unterschiedliche Erziehung. Zum einen stammen beide aus kulturell unterschiedlichen Familien und zum anderen haben die Verhältnisse der Zeit einen anderen Prägungsgrad auf sie, weshalb sie auch eine differenzierte Weltanschauung haben. Das folgende Zitat dient als Beispiel.

Jenö sah wieder blinzelnd in den Himmel. Ob ich Tabak hätte?

„Hör mal“" sagte ich, ,ich bin doch erst neun“.

„Na und“ sagte Jenö, „,ich bin acht“. (Schnurre 2014: 240)

Ebenso unterscheiden sich auch die Essgewohnheiten voneinander stark, während für Jenö Meerschweinchen und Igel zum Tagesmenü gehören, sind diese Tiere für Bruno zu nett, um sie zu essen (Schnurre 2014: 241). Doch diese Gewohnheiten stören Bruno nicht weiter, jedoch die Gewohnheit etwas zu stehlen passt Bruno, im Gegensatz zu seinem Vater, gar nicht (Schnurre 2014: 241). Doch schnell wird er eines Besseren belehrt und versteht den Brauch beim Stehlen. Der Vater ist in der Geschichte recht zuvorkommend und verständnisvoll, was eigentlich den Umständen und der 
traditionellen Vaterfigur der Zeit widerspricht. Während er noch zu Beginn bezüglich der Freundschaft mit einem Zigeunerjungen aufgrund der gesellschaftlichen Normen noch Bedenken hat, verwirft er diesen Gedankengang sehr schnell und ist der Ansicht, dass Bruno alt genug ist, um selbst seine Freunde zu wählen.

\author{
Vater hatte Bedenken, als ich ihm von Jenö erzählte. \\ „Versteh mich recht“, sagte er „,ich hab’ nichts gegen Zigeuner; bloß“ \\ „Bloß” fragte ich. \\ "Die Leute" sagte Vater und seufzte. Er nagte eine Weile an seinen Schnurrbartenden \\ herum. „Unsinn”, sagte er plötzlich, „schließlich bist du jetzt alt genug, um dir deine \\ Bekannten selbst auszusuchen." (Schnurre 2014: 241)
}

Auch aus heutiger Sicht gibt es sicherlich viele Familien, die der Meinung wären, dass ein neun jähriger Junge nicht über diese Entscheidungsfreiheit verfügen sollte und die Eltern darüber entscheiden sollten, wer gut bzw. schlecht für ihr Kind ist. Auch dem Druck der Gesellschaft steht der Vater standhaft gegenüber und ließ es den Kindern nicht anmerken, dass es bezüglich ihrer Freundschaft Beschwerden gab.

\footnotetext{
Später stellte sich heraus, es verging kein Tag, daß sich die Hausbewohner nicht beim Blockwart über Jenös Besuch beschwerten, sogar zur Kreissitzung ist mal einer gelaufen. Weiß der Himmel, wie Vater das jedesmal abbog; mir hat er nie was davon gesagt. (Schnurre 2014: 244)
}

Dieses Schutzverhalten in einer von der Gesellschaft abhängigen Lebensweise vorlegen zu können, passt zum klassischen Vaterbild der Zeit nicht. Auch kristallisiert sich die antiautoritäre Vaterfigur heraus. Bruno, der eben sitzengeblieben war, lernte eine Menge, aber nicht von der Schule, sondern von seinem Freund Jenö (Schnurre 2014: 244). Dieses muss auch sein Vater bemerkt haben, weshalb er auch den Kontakt und die Freundschaft erlaubte. Es geht sogar darüber hinaus, er unterstützte die Freundschaft, indem er oftmals Bruno um Verständnis für Jenös Verhalten bat. Den Umständen der Zeit entsprechend war dem Vater eventuell auch bewusst, dass diese Freundschaft auf begrenzte Zeit sein wird. Als die SA und SS die Bekannten und Familienmitglieder von Jenö im Lastwagen verfrachtet haben, war es Bruno nicht bewusst, was mit ihnen geschehen würde. Nur die Großmutter und die übrigen Alten darunter, wussten was mit ihnen geschah. Bruno war lediglich zu diesem Zeitpunkt, d.h. mit neun Jahren traurig, dass sein Freund weg war. Jetzt als Erwachsener weiß er, was damals passiert ist. Aus diesem Grund wusste auch der Vater, dass den Kindern nicht viel Zeit zusammen verbleiben wird und die Zeit, die ihnen verblieb, sollten sie genießen.

\title{
3.2 Der Brötchenclou
}

Diese Geschichte spielt zu einer Zeit, in der viele Fabriken geschlossen wurden, weil die produzierten Waren im Regal aufgrund Geldmangels nicht gekauft werden konnten. 
Deshalb verloren viele Arbeiter ihre Stellen wie auch Brunos Vater. Diese Situation stört den Familiensegen, wie in vielen Familien der Zeit. Ein Rummel als Abwechslung zum Alltag kam deshalb zum rechten Zeitpunkt. Wer hätte wissen können, dass genau hier eine neue Stelle den Vater erwartet. Mit Bedenken nahm er die Arbeit als Bändiger von Störenfrieden an. Der Grund für seine Bedenken war ein moralischer, denn „,[es] wäre ein Unterschied [...], ob man sich spontan oder auf Bezahlung empörte (Schnurre 2014: 58). Doch die Umstände der Zeit leiteten ihn dazu, die Arbeit anzunehmen. Während den Vater existenzielle Sorgen plagten, schweiften Brunos Gedanken über den baldigen Geburtstag seines Vaters. Bruno wollte ihm eine Freude machen und zu seinem Geburtstag eine Ananas schenken (Schnurre 2014: 59). Dieses Verhalten wird in der traditionellen Rollenverteilung nicht erwartet. Meistens sind es die Eltern und nicht die Kinder, die Geschenke machen. Betrachtet man dieses Verhalten genauer, wird deutlich, dass Bruno trotz seines Alters den Nöten der Zeit sowohl versteht als auch spürt und ihnen entgegenwirken will. Bei diesem Versuch schadet er jedoch seiner eigenen Gesundheit, was auch sein fürsorglicher Vater wohl auch bemerkt und ihn zum Essen zwingt (Schnurre 2014: 60). Dass gerade diese väterliche Fürsorge Bruno einen Strich durch die Rechnung macht, ist doppelt tragisch. Während dem Vater das Wohlbefinden seines Sohnes wichtig ist, ist für den Sohn das Wohlbefinden seines Vaters wichtig. Beide denken an das Wohl des anderen und opfern dafür ihr eigenes Wohlbefinden. So versuchen beide Vater und Sohn sich um das Wohlbefinden des Anderen zu kümmern und bemerken dabei nicht, dass das Gegenteil der Fall ist. Als Bruno die Brötchenwette verlor, machte ihn das so wütend und traurig, doch sein Vater war es, der ihm in dieser schwierigen Situation Beistand. Der Auftritt wird so dargestellt, als ob ein Held aus der sich belustigenden Menge empor hebt und Bruno rettet. „Plötzlich erhob sich jemand im Zuschauerraum und kam langsam nach vorn. Ich fuhr mir über die Augen, und da war es Vater." (Schnurre 2014: 64). Die Menschenmenge, die repräsentativ für die damalige Gesellschaft steht, hatte kein Mitleid mit Bruno und lachte über ihn erbarmungslos. „Sie wollten sich totlachen darauf; sie schlugen sich auf die Schenkel, sie klatschten und schrien wie die Wahnsinnigen." (Schnurre 2014: 64). Der Versuch vom Vater, der ganzen Situation Klarheit zu schaffen, ermutigte sie nur noch mehr in ihrem Verhalten. Kein Scharm und kein Mitleid sind den Menschen geblieben. Wenn doch nur jemand in der Menge wäre, der sich wie einst der Vater über die Menschen empören würde und für Ruhe sorgen würde. Doch nun war es er selbst und sein Sohn, die gepeinigt wurden.

\subsection{Die Leihgabe}

In dieser Geschichte werden dem Leser die schwierigen Verhältnisse der Zeit vor Augen geführt, wobei es diesmal ganz besonders hart für Vater und Sohn ist, denn es ist kurz vor Weihnachten. Das notwendige Geld für die Festlichkeiten fehlt, weil der Vater arbeitslos ist. „Am meisten hat Vater sich jedes Mal zu Weihnachten Mühe gegeben [denn] auf Weihnachten lebte man zu“ (Schnurre 2014: 80). Trotz den vorherrschenden schwierigen Verhältnissen, gab sich der Vater Mühe nicht seine Traurigkeit zu zeigen, dessen sich auch Bruno bewusst ist. Als Vorbild versuchte er seinem Sohn den 
eigentlichen Sinn von Weihnachten zu übermitteln. „Weihnachten [...] wäre das Fest der Freude, das Entscheidende wäre jetzt nämlich nicht traurig zu sein, auch dann nicht, wenn man kein Geld hätte.“ (Schnurre 2014: 80). Für Bruno waren es tröstende Worte und er versuchte seinem Vater die Sorgen zu nehmen, indem er so tat, als ob er sich sonderlich für Weihnachten interessieren würde. Immer wenn sein Vater dabei war, sah er nicht in die geschmückten Schaufenster, doch das zog noch mehr Aufmerksamkeit, weshalb er es auch bald unterließ (Schnurre 2014: 80). Für Bruno hatte es sein Vater schwerer, denn er hatte keinen Vater ,der ihm dasselbe sagen konnte was er [ihm] immer sagte“ (Schnurre 2014: 80). Die Situation gab dem Vater keine Ruhe, sodass er sich entschloss den schönsten, besten Weihnachtsbaum, trotz den Umständen zu beschaffen. Wie er dies anstellen würde, stand noch in den Sternen, doch auf den Rat seiner Gefährtin einen zu klauen, reagierte er sehr empört. Seinen Wertevorstellungen entsprach es nicht und er wollte auch für seinen Sohn vorbildlich sein (Schnurre 2014: 85). Statt einen zu klauen, entschied er sich einen zu borgen. Dieses vorbildliche Verhalten brachte Bruno auf die Idee, sein Glück beim Pfandleiher zu probieren und ihr eigenes zuvor verpfändetes Grammophon auszuleihen, um so seinem Vater zu danken (Schnurre 2014: 87). Diese Geste zeigt, wie einfühlsam und verständnisvoll Bruno trotz seines jungen Alters ist. Der Vater war sehr gerührt und stolz darüber, dass Bruno den Sinn bzw. Unterschied zwischen Borgen und Stehlen verstanden hat (Schnurre 2014: 90). Denn auch wenn die Zeitverhältnisse schwierig waren, wählte der Vater nicht den einfachen, sondern den richtigen Weg.

\subsection{Die merkmalspezifischen Handlungsweisen von Vater und Sohn}

Wolfdietrich Schnurre verbindet in den Vater-Sohn-Geschichten Sozialkritik und Poesie miteinander und gab ein Bild von Berlin sowie dem Lebensgefühl der damaligen Zeit (Walter 1959). Er versetzte seine Figuren in eine Konfliktsituation, welche sie zu überwinden haben und zwar durch ihre innige Verbundenheit. Die politische Rechtsstruktur, der soziale Wandel, Hunger und Arbeitslosigkeit bilden den Ausgangspunkt der schwierigen Situation. Vater und Sohn setzten sich immer wieder sowohl für andere als auch für sich selbst ein, indem sie durch ein gewissenhaftes und verantwortungsvolles Handeln vorbildlich agieren, um aus der Notsituation herauszukommen (Bauer 1996). Dieser Handlungsstrang bildet das Grundgerüst der Kurzgeschichten. In Jenö war mein Freund ist es der soziale Wandel d.h. die Freundschaft mit einem Zigeunerjungen sowie die damalige Rechtsstruktur, die Vater und Sohn in eine Konfliktsituation bringen. In den Kurzgeschichten Der Brötchenclou und Die Leihgabe werden wiederum die Arbeitslosigkeit und Hunger thematisiert. Nach einer ausgiebigen Untersuchung sämtlicher Kurzgeschichten in dem Sammelband Als Vaters Bart noch rot war - Ein Roman in Geschichten fiel die Auswahl auf die zuvor genannten Geschichten, weil sie zum einen in der Weltliteratur bekannt sind und zum anderen die Verbundenheit zwischen Vater und Sohn am deutlichsten hervorheben. Um Wiederholungen zu vermeiden und den Rahmen der Arbeit nicht zu sprengen, wurde der Untersuchungsgegenstand der vorliegenden Arbeit auf diese drei Kurzgeschichten eingegrenzt. Vater und Sohn besitzen eine merkmalspezifische Handlungsweise, die 
sich folgendermaßen zusammenfassen lässt. In den Geschichten wird der Leser mit einer Vaterfigur konfrontiert, die verständnisvoll ist und ein ausgeprägtes Gerechtigkeitsgefühl hat. Die Akzeptanz der Freundschaft zwischen Jenö und Bruno, sowie der Beistand, als Bruno auf der Bühne gepeinigt wurde, weil er die Brötchenwette verlor, zeigen, wie verständnisvoll und beschützend der Vater gegenüber seinem Sohn ist. Jedes Mal handelt er gegen den Druck der autoritären Gesellschaft. Er fungiert wie eine Art Schutzschild für seinen Sohn. Durch diesen Schutz kann sich sein Sohn frei entfalten. Ihm ist es außerdem wichtig, seinem Sohn den Unterschied zwischen Richtig und Falsch sowie zwischen Schwer und Einfach zu zeigen. Insbesondere in der Kurzgeschichte Die Leihgabe wird dieses Handeln deutlich. Er widersetzt sich den zeitgenössischen Schwierigkeiten und versucht trotz des Geldmangels das Weihnachtsfest zu feiern. Hierbei möchte er seinem Sohn die wahre Bedeutung von Weihnachten übermitteln und versucht ein Vorbild $\mathrm{zu}$ sein, indem er nicht den einfachen Weg wählt und einen Weihnachtsbaum stiehlt, sondern sich einen aus dem Wald borgt. Eine richtige Werteerziehung ist für die Vaterfigur wichtig, auch wenn es gegen die zeitgenössische Wertevorstellung entspricht. Oftmals wird in den Kurzgeschichten erwähnt, dass Bruno als Kind nicht die damalige Situation nachvollziehen konnte, doch nun aus dem Blickwinkel eines Erwachsenen sich besser in die Handlungsweise seines Vaters hineinversetzten kann. Durch den Rückblick in die Vergangenheit erst, bemerkt Bruno, wie dankbar er für seine Kindheit sein muss. Die Wahl dieser Erzählperspektive wurde von Schnurre bewusst gewählt, denn dadurch konnte er dem Leser einen dualen Blickwinkel geben. Der Blick eines Kindes aus einem Erwachsenen auf die Gesellschaft, aus der Zukunft in die Vergangenheit. Bruno handelt in den Geschichten stets mit einer Reife, die von einem achtjährigen kaum erwartet wird. Er unterstützt seinen Vater auf eine kindliche und naive Weise die schwere Zeit zu überstehen, indem er z.B. in der Kurzgeschichte Die Leihgabe nicht in die beschmückten Schaufenster hinsah, damit sein Vater nicht traurig wurde. Das Glück seines Vaters ist ihm wichtig, wichtiger als sein eigenes Glück. An der Brötchenwette nahm Bruno teil, um seinem Vater eine Ananas zu schenken, denn dieser hatte bald Geburtstag. In den Geschichten wird der Leser mit einem Kind konfrontiert, das sich nicht über die zeitgenössischen Schwierigkeiten beklagt und es versucht das beste aus der Situation zu machen. Schnurre erschafft eine Figur, die als Kind viel erwachsener ist als die meisten Erwachsenen der Zeit. Zusammenfassend lässt sich folgender Handlungsstrang erkennen. Ausgangspunkt der Geschichten ist eine schwierige Situation, in die Vater und Sohn geraten. In diese Situation gelangen sie aufgrund der bereits erwähnten zeitgenössischen Schwierigkeiten, in der insbesondere die autoritäre Gesellschaft eine antreibende Rolle spielt. Durch ein verständnisvolles und einfühlsames Handeln, schaffen Vater und Sohn die verzwickte Situation zu lösen. Am Ende der Geschichten findet oftmals eine Reinigung des Lesers statt, indem ihm vor Augen geführt wird, wie trotz menschlicher Schwächen der richtige Weg begehen werden kann (Bauer 1996). Die Kurzgeschichten können auch als eine Art Wegweiser gesehen werden, die zeigen, wie entgegen der Zeit und autoritären Gesellschaft gehandelt werden kann. 


\section{Schlussfolgerung}

Die exemplarische Untersuchung der Vater-Sohn-Geschichten zeigte, dass merkmalspezifische Handlungsweisen vorhanden sind, die zur Überwindung von sozialpolitischen Problematiken notwendig waren. In den Kurzgeschichten befinden sich Vater und Sohn in ärmlichen Verhältnissen und repräsentieren somit einen Großteil der damaligen Gesellschaft. Aufgrund der hohen Arbeitslosigkeit leben die Menschen in Armut, d.h. sie können sich ganz einfache lebensnotwendige Dinge nicht leisten. Ausgangspunkt ist diese schwierige Situation. Hinzu kommt der Druck der autoritären Gesellschaft. Die finanzielle Not treibt Vater und Sohn in eine Konfliktsituation, aus welcher sie sich durch den Zusammenhalt sich zu retten versuchen. Das gegenseitige Verständnis von Vater und Sohn hilft ihnen die schwierige Situation zu ertragen und zusammen zu überwinden. Es werden dem Leser zwei Gesichter präsentiert, die wiederum ein weiteres in sich tragen. Der Vater trägt das Gesicht der Vergangenheit und der Sohn das der Zukunft. Diese Dualität lässt sich unter dem Janus-Motiv zusammenfassen. Janus stammt aus dem lateinischen Wort Ianus und bezeichnet den römischen Gott des Anfangs und Endes (Roscher, 1894). Die Vergangenheit symbolisiert das Ende von etwas und die Zukunft den Anfang, doch ohne die Vergangenheit gäbe es keine Zukunft und umgekehrt. Aufgrund dieser komplementären Beziehung zueinander, müssen sie als Ganzes gesehen werden. Dieses Motiv lässt sich auch in den Kurzgeschichten wiederfinden. Die Vaterfigur steht dem Druck der autoritären Gesellschaft entgegen. Er agiert als verständnisvoller und führsorglicher Vater. Trotz den schwierigen Umständen entsprechend verfällt er nicht in die Rolle des traditionellen Vaters und handelt diesem entgegengesetzt. Er unterstützt die Freundschaft mit Jenö dem Zigeunerjungen, steht seinem Sohn in schwierigen Situationen bei, auch wenn der gesellschaftliche Druck noch so stark ist und versucht seinem Sohn auch in schwierigen Zeiten ein Vorbild zu sein. Der Sohn hingegen handelt trotz seines jungen Alters mit einer Reife und einem Verständnis, welches vom Leser nicht erwartet wird. So schafft Schnurre durch die Erzählsituation den Leser zum Nachdenken zu bringen. Die naive, zukunftsorientierte Erzählperspektive durch die Augen von Bruno hat zur Folge, dass dem Leser eine andere Perspektive gezeigt wird. Auch Vater und Sohn stehen in einer komplementären Beziehung zueinander. Das gegenseitige Verständnis zueinander trägt dazu bei, dass schwierige Situationen ertragen werden können. Der Rückblick am Ende der Erzählungen zeigt dem Leser, welche Spuren das Verhalten des Vaters beim Sohn hinterlassen haben. Auch wenn er als Kind manchmal nicht so ganz verstanden hat, wieso und warum sein Vater jenes oder dieses tat, weiß er als Erwachsener jetzt die Zeit mit seinem Vater zu schätzen. Wie der Weihnachtsbaum in der Kurzgeschichte Die Leihgabe ist der Sohn gedeiht und gewachsen, weil sein Vater ihn wohlbehütet erzogen hat und ihm wichtige Wertvorstellungen im Leben mitgegeben hat. Diese starken Wurzeln, in Form von Werten, gaben ihm die Kraft, die schwierigen Verhältnisse der Zeit zu überwinden und nicht mit dem Strom der autoritären Gesellschaft zu schwimmen, sondern ihm entgegen zu schwimmen. Wolfdietrich Schnurre sah in seinem Schreiben die einzige Form der Sühne. Der Mensch ist in seinen Augen schuldig und hat die Aufgabe, sich in seiner 
Schuld zu bekennen, um so seine Schuld auszugleichen. In seinen Geschichten appellierte er an seine Leser, indem er ihnen durch seine sozialkritische Schreibweise, die Schwierigkeiten der Zeit vor Augen führte und ihnen Überwindungswege zeigte. Er wollte ihnen Mut machen. Mut sich der autoritären Gesellschaft, der Rechtsstruktur und den zeitgenössischen Schwierigkeiten entgegenzusetzen. In den Vater-SohnGeschichten werden die bestehenden Normen der damaligen Zeit von den Augen des Kindes aus betrachtet. Es überliefert eine andere Sichtweise auf die Verhältnisse der Zeit und deren Normen. Durch eine reife Handlungsweise belehrt es nicht nur seinen Vater, sondern zugleich auch den Leser. Die traditionelle Vater-SohnKonfliktbeziehung hebt sich in den Geschichten von Schnurre auf und wird durch eine verständnisvolle ersetzt. Die Erziehung und Belehrung von Vater und Sohn stehen nämlich in einer Wechselbeziehung zueinander, d.h. das Zusammenhalten ermöglichte es ihnen die schwierigen Situationen zu überwinden und sich in ihrer Persönlichkeit weiterzuentwickeln. Die konfliktfreie Beziehung zwischen Vater und Sohn, aufgrund lehrreicher Handlungsweisen, geben dem Leser die Möglichkeit, die vorherrschenden gesellschaftlichen Normen zu revidieren. Aus diesem Grund sind die Vater-SohnGeschichten von Wolfdietrich Schnurre pädagogisch wertvoll. Im Folgenden sollen weitere Werke kurz aufgeführt werden, die diese Thematik behandeln, um Anregungen zu weiteren Untersuchungen zu geben.

Wolfdietrich Schnurre behandelt diese Thematik auch in seinen Werken Verrat (1958) und Man sollte dagegen sein (1959). Der Verrat in der gleichnamigen Kurzgeschichte liegt darin, dass der Vater gegen ihre Menschheitsideale handelt, um seinen Sohn am Leben halten zu können. Danach tragen die Schuldgefühle dieses Verrates Vater und Sohn, als Vertreter zweier Generationen zusammen auf ihren Schultern.

Ebenso handelt der Vater in der Geschichte Man sollte dagegen sein dem zeitgemäßen Verständnis wider, indem er nicht der gleichen Ansicht ist wie der Lehrer. Kinder sollten seinem Menschheitsideal zu folge keine Waffen bei sich tragen. Dies tut er allerdings auf eine unkritische und indirekte Weise, um so die Autorität des Lehrers nicht in Frage zu stellen.

\section{Literaturverzeichnis}

Barner, Wilfried (1994): Geschichte der deutschen Literatur von 1945 bis zur Gegenwart. 2. Aktualisierte und erweiterte Auflage. München: C.H. Beck Verlag.

Bauer, Iris (1996): „Ein schuldloses Leben gibt es nicht“ - Das Thema „Schuld“ im Werk von Wolfdietrich Schnurre. 1. Aufl. Paderborn: Igel Verlag.

Bohn, Volker (1995): Deutsche Literatur seit 1945 - Texte und Bilder. Frankfurt am Main. Suhrkamp Verlag.

Brauneck, Manfred (1995): Autorenlexikon deutschsprachiger Literatur des 20. Jahrhunderts. Hamburg: Rowohlt Verlag.

Ernst, Peter (1993): Neues Handbuch der deutschsprachigen Gegenwartsliteratur seit 1945. München: Deutscher Taschenbuch Verlag. 
Hirschenauer, Rupert/ Weber, Alfred (Hrsg.) (1970): Interpretationen zu Wolfdietrich Schnurre. Interpretationen zum Deutschunterricht. München: Oldenbourg Verlag.

Kargı, Birkan (2009): Wolfdietrich Schnurre'nin ,Karşı Çıkmak Gerekiyordu 'Kitabını Oluşturan Kısa Hikâyelerin Savaş Sonrası Alman Edebiyatında Rolü. Çankaya Üniversitesi Fen-Edebiyat Fakültesi, Journal of Arts and Science. Heft 11. 23-48.

Kassel, Dieter (2010): „Er war politisch durch und durch“ https://www.deutschlandfunkkultur.de/er-warpolitisch-durch-und-durch.954.de.html?dram:article_id=145545 (letzter Zugriff:12.04.2020).

Lüdemann, Gunst (1975): Literaturkunde - Dichtung- Wesen und Aussage. Wolfenbüttel: Heckners Verlag.

Richter, Hans Werner (1948): Der Skorpion. Jahrgang 1 Heft 1. München: Wallenstein Verlag.

Roscher, Wilhelm Heinrich (1894): Ianus. Wilhelm Heinrich Roscher (Hrsg.): Ausführliches Lexikon der griechischen und römischen Mythologie. Band 2,1, Leipzig. Sp. 13-55.

Schnell, Ralf (2001): Deutsche Literatur nach 1945 - Aporien des lyrischen Kahlschalgs. Deutsche Literaturgeschichte - Von den Anfängen bis zur Gegenwart. 6. Verbesserte und erweiterte Auflage. Stuttgart; Weimar: Metzler Verlag.

Schnurre, Marina (2014): Wolfdietrich Schnurre - Als Vaters Bart noch rot war - Vater und Sohn Geschichten. Berlin: Berlin Verlag.

Walter, Jens (1959): Poetische Sozialkritik und Selbstverleugnung. Die Berlin gewidmeten Geschichten eines Berliner Autors über Berlin und die kleinen Leute. Die Zeit. Hamburg. 20.03.1959. 